\title{
Letter From the Guest Editor
}
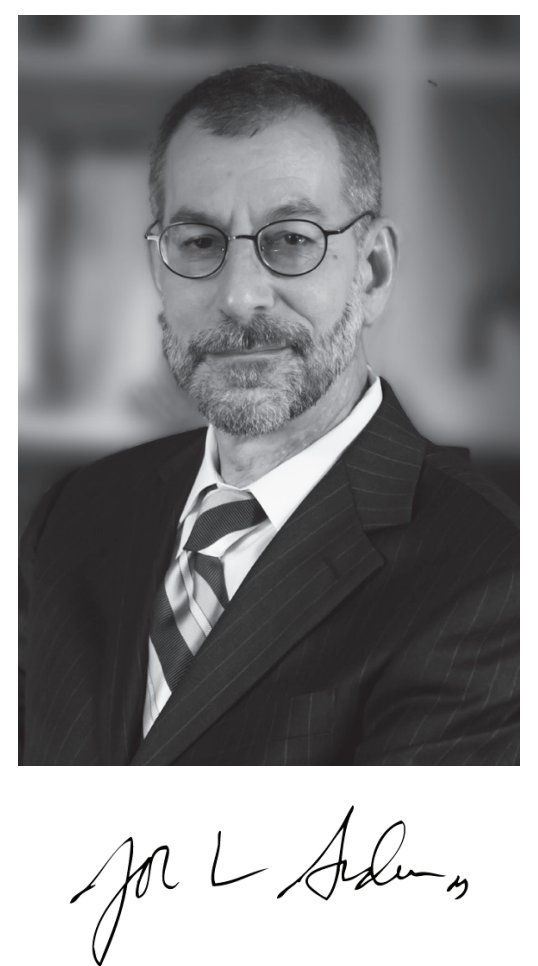

Jonathan L. Arden MD

Guest Editor

I am honored to have been invited to be the Guest Editor of this issue of Academic Forensic Pathology. The theme we have chosen to address in this issue is an examination of selected aspects of postmortem changes and the estimation of time of death. A critical, basic aspect of investigating deaths and medically examining decedents is the recognition of which findings occurred postmortem, the ability to distinguish those from the manifestations of antemortem processes that represent actual disease or injury, and of course, ultimately to be able to determine what caused the death of the person in question. Related to the correct interpretation of postmortem changes is the desire to apply them to a reasonable estimation of the time of death. These issues are frequently of concern and importance to families of decedents, but may also be a crucial factor in deriving an accurate explanation of the circumstances surrounding a death, with implications including assigning responsibility for causing death in a criminal context. Despite the obvious necessity of making such determinations, the evaluation of postmortem changes may be fraught with difficulty, given the many variables that may not be known with precision for a specific death. This has been a conundrum in medicolegal death investigation since its inception.

In this issue we present a range of invited papers that address the topic of postmortem changes as they apply to various special situations, of which I shall highlight a few examples. We examine the process of decomposition as seen in bodies recovered from water, as well as the potential pitfalls of neck dissections. A paper on postmortem computed tomography interpretation describes a special technique being applied to postmortem examination that currently is only available in selected centers, but which clearly will become employed routinely in the future practice of forensic pathology. Another paper is devoted to a different special examination technique, namely the anthropological approach to assessing fractures in deaths with 
substantially greater postmortem intervals. The use of vitreous humor to estimate postmortem interval is reviewed with both an historical perspective and a view toward its potential use in the future. Finally, we offer another historical treatment of the literature on postmortem changes in the 19th century.
I thank the Editor-in-Chief and the Publisher for this opportunity to participate in producing this issue of the Official Publication of the National Association of Medical Examiners. I hope that the contents will be interesting, informative, and especially useful to the readers in their practice of forensic pathology. 